\title{
Existence of Collateral Equilibrium without Survival Assumption ${ }^{\dagger}$
}

\author{
Jaime Orrillo*
}

\section{Resumo}

Este trabalho mostra a existência de um equilíbrio com garantias, sem assumir nenhuma hipótese sobre a positividade estrita, seja das dotações iniciais dos indivíduos (incluindo as ex-post) ou da dotação inicial agregada. Devido a esta falta de positividade estrita das dotações iniciais da sociedade, não temos um equilíbrio. Em vez disso, temos um quase-equilíbrio. Então, apelando ao conceito de irredutibilidade (introduzida por McKenzie em 1959), o qual é adaptado ao modelo com garantias, mostra-se que o quase-equilíbrio é, de fato, um equilíbrio legítimo.

\section{Palavras-Chave}

mercados incompletos, colateral exógeno, oportunidade de arbitragem, hipótese de sobrevivência

\begin{abstract}
This paper shows the existence of a collateral equilibrium without assuming any hypotheses on the strict positivity, be they individual initial endowments, including the ex post initial endowments, or aggregated initial endowments. Because of dropping the strict positivity of social initial endowment we fail to get an equilibrium. Instead, we get a quasi-equilibrium. Then, appealing to the concept of irreducibility (introduced by McKenzie in 1959) which is adapted to the collateral model, we show that the quasi-equilibrium is indeed a legitime equilibrium.
\end{abstract}

\section{Keywords}

incomplete markets, exogenous colateral, arbitrage opportunity, survival assumption

\section{JEL Classification}

D52

+ It is useful to note that the meaning of the word "survival" in this article is related to the positivity of consumers' initial endowments and not to their staying or expulsion from the market which depend on their beliefs, as studied by Sandroni 2000. The author acknowledges CNPq for financial support 304663/2007-7. The author is indebted to an anonymous referee for very helpful suggestions, particularly with regard to exposition of the paper.

* Catholic University of Brasilia. Contact adress: SGAN 916 - Módulo B. ZIP Code: 70790-000 - Asa Norte Brasília - DF. E-mail: orrillo@pos.ucb.br, jjoc@impa.br.

(Recebido em novembro de 2009. Aceito para publicação em setembro de 2010). 


\section{Introduction}

The word "survival" implies the existence of some event that causes hurt or the loss of something that prevents some activity after the catastrophe passes (catastrophe, in this case, means the lack of future income of all the agents). The classic models of general equilibrium with uncertainty assume that agents have strictly positive initial endowments including the ex post initial endowments (or its more weaker version which declares the aggregated initial endowments to be strictly positive). Strict positivity of initial endowments of all the agents is fundamental to guarantee the existence of equilibrium. However, in the real world there is no one or nothing that guarantees agents will have some income allowing them to trade after uncertainty is solved, unless there is some mechanism which allows the transfer of positive wealth from one period to another. This mechanism could be financial markets. However, such financial institutions do not guarantee that the ex post wealth is at least non-negative, since the ex post financial income (due to asset returns) might be negative enough to create a non-positive total income. In this paper we will analyze such transfers in a GEI model by allowing agents to default on their promises. For this reason we require from borrowers to put aside collateral which will assume to be durable as in Geanakoplos and Zame (2002, 2007') (henceforth GZ).

Collateral, as a means to enforce promises, has been analyzed in the context of general equilibrium with incomplete markets by GZ $(2002,2007)$. They stress the importance of requiring collateral from borrowers each time the latter sell assets, stating that this has profound impacts on the whole economy even when there is no default). ${ }^{2}$ In the GZ paper several examples are offered which illustrate diverse situations, from consumption distortion to the effect on the volatility of prices of both assets and commodities.

The objective of this paper is to show existence of equilibrium without assuming any assumption on the strict positivity of the initial endowments including the ex post initial endowments or aggregated initial endowments. That is, the survival assumption, which states that all individuals who have either strictly positive initial endowments or initial social endowments belong to the interior of the consumption set, is dropped. Due to this fact we do not obtain an equilibrium but rather a quasi-equilibrium.

We adapt the notion of quasi-equilibrium for a collateral economy in the same way that Gottardi and Hens (1996) do for the case of a standard incomplete market model. They proved the existence of equilibrium in a GEI model with numeraire

1 Version more recent.

2 For a broad discussion on collateral, we refer to Geanakoplos (1997). 
assets while still assuming that at least one agent has strict positive initial endowments in at least one state of nature. They use the concept of irreducibility according to McKenzie (1959) to redistribute income among all the agents so that a quasi-equilibrium becomes a legitimate equilibrium. We also use this concept of irreducibility adapted to the collateral model to get our result.

However, it is useful to stress that, contrary to Gottardi and Hens (1996), we do not assume strict positivity of initial endowment of any agent. To be more precise, all agents, in this paper, are assumed to have second-period initial endowments equal to zero implying contingent income zero for all agents. Besides the fact that it is not very realistic to assume that at least one agent has positive wealth in an environment with uncertainty, it also provokes a serious technical problem in the agents' budget correspondence, namely, they fail to be lower hemi continuity. However, in the collateral model this problem disappears due to depreciation structure of goods assumed here.

The methodology used to show the existence of equilibrium is as follows: first we slightly modify the initial endowments of our original collateral economy so that in the new parameterized economy all the hypothesis imposed by GZ $(2002,2007)$ are satisfied. So our parameterized economy has an equilibrium. Next, by a limit process we show that the allocation of the limit economy is a quasi-equilibrium supported by a non-zero commodity-asset price system. We then use the fact that the depreciation of the first-period social endowment is strictly positive, like in GZ (2002, 2007) together with the market clear conditions to show that at least one agent has a strictly positive income. Since the agents' utility functions are strictly increasing the above agent with positive income will be maximizing his/her utility in the limit economy. Also, all the prices in the limit economy are strictly positive. Finally, to reach equilibrium in the limit economy we use the concept of irreducibility, in the context of a collateral economy, introduced by McKenzie.

The plan of the paper is as follows: in section 2 we describe the model by stating the hypotheses and defining equilibrium, quasi-equilibrium and the concept of irreducibility in our setting. In it we also establish the determinateness of individual behavior and define the arbitrage condition. Section 3 is dedicated to the proof of the existence of both quasi-equilibrium and equilibrium in the collateral economy. Section 4 offers some conclusions. We end with an appendix containing a miscellaneous of notations. 


\section{The Model}

We shall follow, for the most part, Geanakoplos and Zame's model (see GZ, 2002) with slight differences in the notation. ${ }^{3}$ To understand our notation, see the beginning of the appendix. We consider a two-period exchange economy with a finite number $H$ of consumers and $L$ commodities. There are $S$ states of nature to be revealed in the second period, and in the first period there is just one state of nature (called state 0 ), in which $H$ agents trade in $L$ commodities and $J$ assets. Default is allowed and the lenders are protected by a collateral structure which is exogenously given and backs short sales issued by the borrowers. More precisely, the collateral is modelled by a physical commodity bundle $C=\left(C_{1}, \ldots, C_{J}\right) \in R_{+}^{L J}$ where the $j-t h$ vector backs the sale of one unit of asset $j \in J$. It is assumed that $C_{j}$ is depreciated according to a state - contingent positive linear transformation, $Y_{s}: R^{L} \rightarrow R_{+}^{L}$. Let us denote by $Y_{s}=\left[Y_{s}^{1} \ldots Y_{s}^{l} \ldots Y_{s}^{L}\right] \in R_{+}^{L \times L}$ the matrix that represents the above positive linear transformation which is $Y_{s}^{l} \in R_{+}^{L}, l \in L$ its $l-$ th column.

Each asset $j \in J$ is characterized by its promise $A^{j} \in R_{+}^{S \times L}$ and by the collateral $C_{j} \in R_{+}^{L}$ that backed it. Agents are characterized by their utility function $U^{h}: R_{+}^{L(S+)} \rightarrow R$ and their first- period initial endowments $\omega^{h} \in R_{+}^{L}$. Since default is permitted, each agent has the option of delivering less than he promised. If we assume that the commodity price system is $p \in R_{+}^{L(S+)}$, the value of the promise, in each state $s$, is $p_{s} A_{s}^{j}$. Let $D_{s}^{h j}$ denote what agent $h$ decides to deliver. As the only consequence of default is the seizure of the collateral, it then follows that any rational borrower will choose to deliver the minimum of the face value and the depreciated collateral value. Similarly, each lender expects to receive only the minimum between the contingent claim and the depreciated collateral value. Thus, the delivery on asset $j$ in the second period is defined as

$$
D_{s}^{j}=\min \left\{p_{s} A_{s}^{j}, p_{s} Y_{s} C_{j}\right\}
$$

Define by $R=R(p, A, C, Y)$ to be the matrix

$$
[p Y D p Y C-D]_{S \times(L+2 J)}
$$

where the sub- matrix $p Y$ is of order $S \times L$ whose $(s, l)$ entry is the number $p_{s} Y_{s}^{l}$. In a similar way $D$ and $p Y C-D$ are the sub-matrixes of order $S \times J$ whose $(s, j)$

3 Actually, the notation comes from the old version of this model, namely Geanakoplos, Zame, and Dubey (1995). 
entries are, respectively, the numbers $D_{s}^{j}$, and $p_{s} Y_{s} C_{j}-D_{s}^{j}$. Denote by $<R>$ the sub-space generated by the columns of the matrix $R$ just defined.

We assume that

- Each agent $h \in H$ has a continuous, concave, and strictly increasing ${ }^{4}$ utility function $U^{h}: R_{+}^{L(S+1)} \rightarrow R$

- $\omega_{o}^{h}>0$ and $\omega_{-o}^{h}=0, \forall h \in H$

To understand the meaning of the sub-index " $-o$ ", see the miscellaneous notation in the appendix.

Notice that we do not require strict positivity of the initial social endowment in contrast to GZ (2002) who do require it.

A price system is a vector $\pi \in R_{+}^{J}$ of securities, and a state-contingent consumption price vector is $p \in R_{+}^{L(S+1)}$.

Definition 1: Given a price system $(p, \pi) \in R_{+}^{L(S+1} \times R_{+}^{J}$ a consumption-portfolio choice $(x, \theta, \varphi) \in R_{+}^{L(S+)} \times R_{+}^{2 J}$ is budget-feasible for agent $h \in H$ if

$$
\begin{aligned}
& p_{o}\left(x_{o}+C \varphi-\omega_{o}^{h}\right)+\pi \theta \leq \pi \varphi \\
& p_{s}\left(x_{s}-Y_{s} x_{o}\right) \leq D_{s} \theta+\left(p_{s} Y_{s} C-D_{s}\right) \varphi, \quad s \in S
\end{aligned}
$$

The budget constraint (1) states that the cost of a net purchase of goods $p_{o}\left(x_{o}+C \varphi-\omega_{o}^{h}\right)$ plus the lending $\pi \theta$ (due to the purchase of assets) cannot exceed the borrowing $\pi \varphi$ (due to sale of assets). The budget constraint (2) tells us that after $s \in S$ occurred at date 1 , the consumer must again decide on his net purchases of goods $p_{s}\left(x_{s}-Y_{s} x_{o}\right)$, which must be financed from receipts of assets that he purchased in the first period and from net deliveries the agent makes on assets he sold.

Consumption-portfolio choices satisfying the budget constraint (1) and (2) define consumer $h^{\prime}$ budget set:

$$
B^{h}(p, \pi)=\left\{(x, \theta, \varphi) \in R_{+}^{L(S+1)} \times R_{+}^{2 J}:(1) \text { and (2) are satisfied }\right\}
$$

4 GZ (2002) makes a weaker hypothesis. 
Definition 2: Given $(p, \pi)$, a budget-feasible choice $(x, \theta, \varphi)$ for agent $h$ is optimal if there is no budget-feasible choice $\left(x^{\prime}, \theta^{\prime}, \varphi^{\prime}\right) \in B^{h}(p, \pi)$ for agent $h$ such that

$$
U^{h}\left(x_{o}^{\prime}+C \varphi^{\prime}, x_{-o}^{\prime}\right)>U^{h}\left(x_{o}+C \varphi, x_{-o}\right)
$$

Remark: The optimality of $(x, \theta, \varphi)$ for agent $h$ can be stated in an equivalent way, as follows:

$$
\begin{aligned}
& \forall\left(x^{\prime}, \theta^{\prime}, \varphi^{\prime}\right) \in R_{+}^{L(S+1} \times R_{+}^{2 J}, U^{h}\left(x_{o}^{\prime}+C \varphi^{\prime}, x_{-o}^{\prime}\right)>U^{h}\left(x_{o}+C \varphi, x_{-o}\right) \wedge \\
& p_{s}\left(x_{s}^{\prime}-Y_{s} x_{o}^{\prime}\right) \leq D_{s} \theta^{\prime}+\left(p_{s} Y_{s} C-D_{s}\right)^{\prime}, \quad s \in S \Rightarrow p_{o}\left(x_{o}^{\prime}+C \varphi^{\prime}\right)+\pi \theta^{\prime}-\pi \varphi^{\prime}>p_{o} \omega_{o}^{h}
\end{aligned}
$$

Since the optimal is budget feasible it follows that

$$
p_{o}\left(x_{o}+C \varphi\right)+\pi \theta-\pi \varphi \leq p_{o} \omega_{o}^{h} .
$$

Therefore the previous implication becomes

$$
\begin{gathered}
\forall\left(x^{\prime}, \theta^{\prime}, \varphi^{\prime}\right) \in R_{+}^{L(S+1} \times R_{+}^{2 J}, U^{h}\left(x_{o}^{\prime}+C \varphi^{\prime}, x_{-o}^{\prime}\right)>U^{h}\left(x_{o}+C \varphi, x_{-o}\right) \wedge \\
p_{s}\left(x_{s}^{\prime}-Y_{s} x_{o}^{\prime}\right) \leq D_{s} \theta^{\prime}+\left(p_{s} Y_{s} C-D_{s}\right) \varphi^{\prime}, \quad s \in S \\
\Downarrow \\
p_{o}\left(x_{o}^{\prime}+C \varphi^{\prime}\right)+\pi \theta^{\prime}-\pi \varphi^{\prime}>p_{o}\left(x_{o}+C \varphi\right)+\pi \theta-\pi \varphi
\end{gathered}
$$

We define an economy as a collection of individuals, a financial structure ${ }^{5}$, and a depreciation structure. In symbols we have

$$
E=\left[\left(U^{h}, \omega_{o}^{h}\right)_{h \in H} ;(A, C) ; Y\right]
$$

It is useful to point out that agents in the economy only have initial endowments at the beginning of the economic activity.

5 Consisting of promises and exogenous collateral. 


\section{Definition 3: (Exogenous Collateral Equilibrium)}

An exogenous collateral equilibrium is a price system $(p, \pi)$, and a collection $\left(x^{h}, \theta^{h}, \varphi^{h}\right)_{h \in H}$ of optimal choices for the respective agents. In addition, given $(p, \pi)$, all markets clear:

$$
\begin{aligned}
& \sum_{h \in H}\left(x_{o}^{h}+C \varphi\right)=\sum_{h \in H} \omega_{o}^{h}, \sum_{h \in H} x_{s}^{h}=\sum_{h \in H}\left[Y_{s}\left(x_{o}+C \varphi^{h}\right)\right], \forall \in S \\
& \sum_{h \in H} \theta^{h}=\sum_{h \in H} \varphi^{h} .
\end{aligned}
$$

Let

$$
\begin{aligned}
& F=\left\{(x, \varphi) \in R_{+}^{L(S+1) H} \times R_{+}^{J H}: \sum_{h \in H}\left[x_{o}^{h}+C \varphi^{h}-\omega_{o}^{h}\right]=0\right. \\
& \left.\wedge \sum_{h \in H} x_{-o}^{h}-Y\left[x_{o}^{h}+C \varphi^{h}\right]=0\right\}
\end{aligned}
$$

be the set of all feasible allocations. Notice that we have put short sales as part of feasible allocation since the demand for durable goods used for collateral depends on short sales.

The question is now to know whether there is a price system for which there exists an equilibrium on $E$. To obtain this we need to make some assumptions based on the characteristics of our economy.

What we will first demonstrate is the existence of a quasi-equilibrium for our collateral economy which is a weaker notion than that of equilibrium. Before providing assumptions for our model we are going to define what a quasi-equilibrium means in our context.

\section{Definition 4: (Exogenous Collateral Quasi-equilibrium)}

An exogenous collateral quasi-equilibrium is a price system $(p, \pi)$, and a collection $\left(x^{h}, \theta^{h}, \varphi^{h}\right)_{h \in H}$ of budget-feasible choices for the respective agents. In addition, given $(p, \pi)$, all markets clear as in Definition 3 and for each agent $h \in H$, we have

$$
\text { for all fixed }\left(x^{\prime}, \theta^{\prime}, \varphi^{\prime}\right) \in R_{+}^{L(S+1)} \times R_{+}^{2 J}, U^{h}\left(x_{o}^{\prime}+C \varphi^{\prime}, x_{-o}^{\prime}\right)>U^{h}\left(x_{o}+C \varphi, x_{-o}\right)
$$


and

$$
p_{s}\left(x_{s^{\prime}}-Y_{s} x_{o^{\prime}}\right) \leq D_{s} \theta^{\prime}+\left(p_{s} Y_{s} C-D_{s}\right) \varphi^{\prime}, \quad \text { for all } s \in S
$$

implies

$$
p_{o} x_{o^{\prime}}+C \varphi^{\prime}+\pi \theta^{\prime}-\pi \varphi^{\prime} \geq p_{o} \omega_{o}^{h}
$$

The only difference between equilibrium and quasi-equilibrium is that any choice which is strictly preferred to $\left(x^{h}, \theta^{h}, \varphi^{h}\right)$ and budget-feasible in each state of nature of the second period must cost at least the value of the first-period initial endowment.

Under (Al) the investor's problem has a solution if, and only if, there are no arbitrage opportunities, see Orrillo (2005). That is, if and only if the following condition is satisfied:

$$
(N A C) \nexists(\theta, \varphi) \in R_{+}^{2 J}: \pi \theta+\left(p_{o} C-\pi\right) \varphi \leq 0 \text { and } R(\theta, \varphi)>0
$$

The following characterization of NAC is given in the following lemma due to Araujo et al. (2005).

Lemma 1: There are no arbitrage opportunities if and only if $\exists \beta \in R_{++}^{S}$ such that

$$
\sum_{s \in S} \beta_{s} D_{s}^{j} \leq \pi_{j}<\sum_{s \in S} \beta_{s} D_{s}^{j}+\left(p_{o} C_{j}-\sum_{s \in S} \beta_{s} p_{s} Y_{s} C_{j}\right)
$$

Notice that (3) above implies

$$
p_{o} C_{j}-\sum_{s \in S} \beta_{s} p_{s} Y_{s} C_{j}>0, \text { and } p_{o} C_{j}-\pi_{j}>0, \forall j \in J
$$

Since utility functions are assumed to be strictly increasing by (Al), commodity prices equal to zero will be ruled out by assumption. We will make the following assumptions which will assure the existence of equilibrium without survival assumption.

- $C_{j} \neq 0, \quad \forall j \in J$.

- $Y_{s}\left(\sum_{h} w_{o}^{h}\right)>>0, \forall s \in S$. 
- $A_{s}^{j} \neq 0, \forall(j, s) \in J \times S ; Y_{s}^{l} \neq 0, \forall(s, l) \in S \times L$

\section{Remarks:}

1. As the collateral is the only method of enforcing promises (A3) is quite natural: any asset which demands no collateral requirement would deliver nothing, and therefore will have zero price in equilibrium. Assumption (A4) assures that in the second period, in any state of nature, the value of first -period initial social endowment ${ }^{6}$ is strictly positive for any price $p_{s}>0$ Now if we want to relax (A4) to become $>0$ instead of $>0$ we would have to impose a stronger structure on $Y_{s}$. Namely, $a Y_{s} \in R_{++}^{L}, \forall a>0$.

2. Assumption (A5) assures the existence of at least one portfolio ${ }^{7}(\theta, \varphi)$ which is always desirable. i.e., a portfolio yielding a non-negative, non-zero pay-off. To see this, notice that $p_{s} \in \Delta_{++}^{L}$ implies that $D_{s}^{j}$ is strictly positive and bounded away from zero. This together with (3) also implies that $\pi_{j}>0$. In fact:

Define the sets $L_{1}=\left\{l \in L: A_{s l}^{j}>0\right\}, L_{2}=\left\{l \in L: C_{j l}>0\right\}$ and $L_{3}^{l}=\left\{l^{\prime} \in L: Y_{s l^{\prime}}^{l}>0\right\}$. These sets are all nonempty by (A3) and (A5) respectively. Since $p_{s} \in \Delta_{++}^{L}$, one easily has that $p_{s} Y_{s} C_{j} \geq \min _{l \in L}\left\{\left(\min _{l^{\prime} \in L_{3}} Y_{s l^{\prime}}^{l}\right)\right\}\left(\min _{l \in L_{2}} C_{j l}\right)>0$. Using the above fact one has that $p_{s} A_{s}^{j} \geq \min _{l \in L_{1}} A_{s l}^{j}>0$. Define $\alpha_{s j}$ to be the minimum between $\min _{l \in L}\left\{\left(\min _{l^{\prime} \in L_{3}} Y_{s l^{\prime}}^{l}\right)\right\}\left(\min _{l \in L_{2}} C_{j l}\right)$ and $\min _{l \in L_{1}} A_{s l}^{j}>0$. This parameter, $\alpha_{s j}$, is strictly positive. From the definition of $D_{s}^{j}$, it then follows that $0<\alpha_{s j} \leq D_{s}^{j}$. This implies that $D_{s} \theta+\left(p_{s} Y_{s} C-D_{s}\right) \varphi>0$ for all $s$. This in turn implies that consumers will never be satiated in their asset demand. See assumptions A.3. in Gottardi and Hens (1996).

3. Let $A$ be the set of all commodity - asset prices $(p, \pi)=\left(p_{o}, p_{1}, \ldots, p_{S}, \pi\right) \in R_{++}^{L} \times R_{++}^{L S} \times R^{J}$ that admit no arbitrage opportunity. That is, those which satisfy Corollary 1 . Denote by $N$ the set

$$
\left\{\left(p_{o}, \pi\right) \in R_{++}^{L} \times R^{J}: \exists p_{s} \in R_{++}^{L}, s \in S \text { such that }(p, \pi) \in A\right\}
$$

If there is no confusion each time that we write $(p, \pi) \in N \times R_{++}^{L} \ldots \times R_{++}^{L}$ it will be understood that $\left(p_{o}, \pi\right)$ will belong to $N$ and the prices $p_{s}$ will belong to $R_{++}^{L-1}$. The prices $p_{s}$ can be assumed to belong to $\Delta_{++}^{L}$ without loss of generality

6 Of course, transformed via $Y_{s}$.

7 Indeed any $(\theta, \varphi) \in R_{+}^{2 J}$ with $\theta \neq 0$. 
since the second-period budget constraint is unchangeable when $p_{s}$ is substituted by $\alpha p_{s}$ for all $\alpha>0$.

4. It is easy to check that for any $p \in R_{++}^{S L}$ the set $N$ is convex. ${ }^{8}$ Thus, so is the set

$$
N \times\left(\Delta_{++}^{L-1} \times \ldots \Delta_{++}^{L-1}\right) \subset R_{++}^{(S+1) L} \times R_{+}^{J}
$$

To abbreviate the notation we will write $\Delta_{++}^{S(L-1)}$ instead of $\Delta_{++}^{L-1} \times \ldots \times \Delta_{++}^{L-1} \subset R_{++}^{S L}$.

It is convenient to decompose the individual budget set of agent $h$ into two sets: Namely, the set

$$
B_{o}^{h}\left(p_{o}, \pi, \omega_{o}^{h}, A, Y\right)=\left\{\left(x_{o}, \theta, \varphi\right) \in R^{L} \times R_{+}^{2 J}: p_{o} x_{o}+\pi \theta+\left(p_{o} C-\pi\right) \varphi \leq p_{o} \omega_{o}^{h}\right\}
$$

is the set of date 0 admissible trades. Note that in this set it is not assumed ${ }^{9}$ that the ex post income is non negative since in the collateral model the ex post financial income is always non-negative. i.e.,

$$
D_{s} \theta+\left(p_{s} Y_{s} C-D_{s}\right) \varphi \geq 0, s=1, \ldots, S .
$$

In a similar way the sets of spot $s$ admissible trades are defined to be

$$
B_{o}^{h}\left(p_{o}, \pi, \omega_{o}^{h}, A, Y\right)=\left\{\left(x_{o}, \theta, \varphi\right) \in R^{L} \times R_{+}^{2 J}: p_{o} x_{o}+\pi \theta+\left(p_{o} C-\pi\right) \varphi \leq p_{o} \omega_{o}^{h}\right\}
$$

The next assumption requires that for all admissible ${ }^{10}$ first-period commodity prices and all asset prices in the closure of $N$, for at least one agent, the set of budget feasible asset trades has a nonempty interior.

(A6) For all $\left(p_{o}, \pi\right) \in \bar{N} \cap\left[\Delta_{+}^{J+L-1}\right], \exists h \in H$ such that the interior of his/her firstperiod budget set $B_{o}^{h}\left(p_{o}, \pi, \omega^{h}, A, Y\right)$ defined by

$$
\left\{\left(x_{o}, \theta, \varphi\right) \in R^{L} \times R_{+}^{2 J}: p_{o} x_{o}+\pi \theta+\left(p_{o} C-\pi\right) \varphi<p_{o} \omega_{o}^{h}\right\}
$$

is nonempty.

8 Indeed $N$ is a cone.

9 In contrast to Gottardi and Hens (1996) who do assume and form part of it.

10 Which excludes prices equal to zero since utility functions are strictly increasing by (Al) . 
Similar to Gottardi and Hens Assumption (A6) guarantees that a minimum wealth condition is always satisfied for at least one agent. For now we are not concerned with the strict positivity of the ex post financial income in a given state because this problem will be treated later in Step 2 of the demonstration of Theorem 1.

To end the description of the model we must assume that the economy is resource related. This assumption demands that any subset of individuals be endowed with resources which can be redistributed to the complementary subset of individuals so as to improve their welfare at any feasible allocation, taking into account the constraints arising from the default, collateral and market incompleteness. ${ }^{11}$

Denote by

$$
\left.P_{2}(H)=\left\{H_{1}, H_{2}\right\}: \phi \neq H_{i} \subset H, i=1,2 \wedge H_{1} \cup H_{2}=H\right\}
$$

the set of all non-trivial partitions of the agents $H$.

\section{Irreducibility Assumption}

We are now ready to establish the notion of irreducibility in our context:

$$
\begin{aligned}
& \text { (A7) } \forall\left\{H_{1}, H_{2}\right\} \in P_{2}(H) \quad \forall\left(x_{-o}^{h}\right)_{h \in H} \in R_{+}^{L S H}, \forall p_{-o} \in \Delta_{++}^{S(L-1)} . \\
& \exists\left(x_{o}^{h}, \theta^{h}, \varphi^{h}\right) \in R_{+}^{L} \times R_{+}^{2 J}:\left(p_{s} x_{s}\right)_{s \in S}=R\left(x_{o}^{h}, \theta^{h}, \varphi^{h}\right), \forall h \in H ; \wedge\left(x_{o}^{h}, x_{-o}^{h}, \varphi^{h}\right)_{h \in H} \in F . \\
& \exists y=\left(y^{h}\right)_{h \in H} \in R^{L(S+1) H} \text { with } y^{h}=\left(y_{o}^{h}, \tilde{y}^{h}\right) \in R^{L(S+1)} \text { such that } \\
& \sum_{h \in H} y^{h}=0 \wedge \tilde{p} \square \tilde{y}^{h} \in<R(p, A, C, Y)>, \forall h \in H \\
& \omega_{o}^{h}+y_{o}^{h} \geq 0, \tilde{y}^{h} \geq 0 \forall h \in H_{1}
\end{aligned}
$$

and $U^{h}\left(x_{o}^{h}+C \varphi^{h}+y_{o}^{h}, x_{-o}^{h}+\tilde{y}^{h}\right) \geq U^{h}\left(x_{o}^{h}+C \varphi^{h}, x_{-o}^{h}\right) \forall h \in H_{2}$

with strict inequality for some $h \in H_{2}$.

Assumption (A7) states that at all relevant prices a minimum wealth condition holds for all agents.

11 Incompleteness in the collateral model is defined the same way as in the standard GEI model with the only difference being that in the collateral model an asset is defined by its promise and the collateral that backs it. 


\section{The Existence Theorem}

Now we are ready to demonstrate our main theorem. We do it in several steps:

Theorem 1: Under Assumptions A1-A7 the economy $E$ has an exogenous collateral equilibrium.

\section{Proof:}

Step 1: Existence of quasi-equilibrium

Define, for all $n \geq 1$ the following economy sequences

$$
E^{n}=\left[\left(u^{h},\left(\tilde{\omega}_{o}^{h n},\left(\tilde{\omega}_{1}^{h n}, \ldots, \tilde{\omega}_{S}^{h n}\right),(A, C), Y\right]\right.\right.
$$

where $\tilde{\omega}_{o}^{h n}=\left(\omega_{o}^{h}+\frac{1}{n} 1\right) \in R_{++}^{L}$, and $\widetilde{\omega}_{s}^{h}=\frac{1}{n} 1 \in R_{++}^{L}, \forall s \in S$. Notice that all the assumptions listed in GZ (2002), p. 10 are satisfied. Thus from GZ (2002) it follows that each economy $E^{n}, n \geq 1$ has an equilibrium. Thus, a price system $\left(p^{n}, \pi^{n}\right) \in N \times \Delta_{++}^{S(L-1)}$ and an allocation of consumption and investments $\left(x_{n}^{h}, \theta_{n}^{h}, \varphi_{n}^{h}\right)_{h \in H}$ exist such that

$$
\sum_{h \in H} x_{o n}^{h}+C \varphi_{n}^{h}=\sum_{h \in H}\left(\omega_{o}^{h}+\frac{1}{n} 1\right), \sum_{h \in H} x_{s n}^{h}=\sum_{h \in H}\left[\frac{1}{n} 1+Y_{s}\left(x_{o n}^{h}+C \varphi_{n}^{h}\right)\right], \sum_{h \in H} \theta_{n}^{h}=\sum_{h \in H} \varphi_{n}^{h}
$$

and $\left(x_{n}^{h}, \theta_{n}^{h}, \varphi_{n}^{h}\right)$ maximize $U^{h}\left(x_{o}+C \varphi, x_{-o}\right)$ subject to

$$
\begin{aligned}
& p_{o}^{n} x_{o}+\pi^{n} \theta+\left(p_{o}^{n} C-\pi^{n}\right) \varphi \leq p_{o}^{n} \omega_{o}^{h}+\frac{\sum_{l \in L} p_{o l}^{n}}{n} \\
& p_{s}^{n} x_{s} \leq \frac{1}{n}+D_{s}^{n} \theta+\left(p_{s}^{n} Y_{s} C-D_{s}^{n}\right) \varphi+p_{s}^{n} Y_{s} x_{o}, s \in S .
\end{aligned}
$$

It is useful to point out that in GZ (2002) the set $N$ does not appear explicitly. The optimality condition of each consumer, in each economy $E^{n}$, implies that the prices $\left(p_{o}^{n}, \pi^{n}\right) \in N$. See Orrillo (2005).

From the remark of Definition 2, the optimality of $\left(x_{n}^{h}, \theta_{n}^{h}, \varphi_{n}^{h}\right)$ is equivalent to the two following conditions: for all $h \in H$ one has 


$$
\begin{aligned}
& U^{h}\left(x_{o}+C \varphi, x_{-o}\right)>U^{h}\left(x_{o n}^{n}+C \varphi_{n}^{h}, x_{-o n}^{h}\right) \text { and } p_{s}^{n}\left(x_{s}-Y_{s}\left(x_{o}+C \varphi\right)\right)=D_{s}^{n}(\theta-\varphi)+\frac{1}{n}, s \in S \\
& \Downarrow \\
& \pi^{n} \theta+\left(p_{o}^{n} C-\pi^{n}\right) \varphi>\pi^{n} \theta_{n}^{h}+\left(p_{o}^{n} C-\pi^{n}\right) \varphi_{n}^{h} \\
& \pi^{n} \theta_{n}^{h}+\left(p_{o}^{n} C-\pi^{n}\right) \varphi_{n}^{h}+p_{o}^{n} \omega_{o}^{h}+\frac{\sum_{l \in L} p_{o l}^{n}}{n}=0 \\
& \text { and } p_{s}^{n}\left(x_{s n}^{h}-Y_{s}\left(x_{o n}^{h}+C \varphi_{n}^{h}\right)\right)=D_{s}^{n}\left(\theta_{n}^{h}-\varphi_{n}^{h}\right)+\frac{1}{n}, s \in S
\end{aligned}
$$

Notice that under Al, Item 2 of remark $^{12}$ of Assumption (A5), (4) and (5) imply the validity of

$$
\begin{aligned}
& \forall s \in S, \\
& U^{h}\left(x_{o n}^{h}+C \varphi_{n}^{h}, x_{1 n}^{h}, \ldots, x_{s n}, \ldots, x_{S n}^{h}\right)>U^{h}\left(x_{o n}^{h}+C \varphi_{n}^{h}, x_{-o n}^{h}\right) \\
& \Downarrow \\
& p_{s}^{n}\left(x_{s}+Y_{s}\left(x_{o}+C \varphi\right)\right)>p_{s}^{n}\left(x_{s n}^{h}+Y_{s}\left(x_{o n}^{h}+C \varphi_{n}^{h}\right)\right)
\end{aligned}
$$

Letting $^{13} n \rightarrow \infty$, from the compactness of the price domain, the non-nullity of collateral and the feasible allocations set one has

$$
\begin{aligned}
& p_{s}^{n} \rightarrow \bar{p}_{s} \in \Delta_{+}^{L-1}, s \in S \\
& \left(p_{o}^{n}, \pi^{n}\right) \rightarrow\left(\bar{p}_{o}, \bar{\pi}\right) \in \Delta_{+}^{L+J-1} \cap \bar{N} \\
& \left(x_{n}^{h}, \theta_{n}^{h}, \varphi_{n}^{h}\right) \rightarrow\left(\bar{x}^{h}, \bar{\theta}^{h}, \bar{\varphi}^{h}\right)
\end{aligned}
$$

where markets clear at the original economy $E$

12 Which concludes that consumers will never be satiated in their assets demand. 13 Passing to a subsequence if necessary. 


$$
\sum_{h \in H}^{-x_{o}^{h}}+C \bar{\varphi}^{-h}=\sum_{h \in H} \omega_{o}^{h}, \sum_{h \in H}^{-x_{s}^{h}}=\sum_{h \in H}\left[Y_{s}\left(\bar{x}_{o}^{h}+C \bar{\varphi}^{h}\right)\right], \sum_{h \in H} \bar{\theta}^{h}=\sum_{h \in H}^{\bar{\varphi}^{h}}
$$

As in Gottardi and Hens (1996), we can take limit (passing to a subsequence if necessary) when $n \rightarrow \infty$ in $^{14}$ (4), (5) and (6). Thus, one has that for all $h \in H$ the following is true:

$$
\begin{gathered}
U^{h}\left(x_{o}+C \varphi, x_{-o}\right) \geq U^{h}\left(\bar{x}_{o}^{n}+C \bar{\varphi}^{h}, \bar{x}_{-o}^{h}\right) \text { and } \bar{p}_{s}\left(x_{s}-Y_{s}\left(x_{o}+C \varphi\right)\right)=\bar{D}_{s}(\theta-\varphi), s \in S \\
\Downarrow \\
\bar{\pi} \theta+\left(\bar{p}_{o} C-\bar{\pi}\right) \varphi \geq \bar{\pi} \bar{\theta}^{h}+\left(\bar{p}_{o} C-\bar{\pi}\right) \bar{\varphi}^{h} \\
\bar{\pi} \bar{\theta}^{h}+\left(\bar{p}_{o} C-\bar{\pi}\right) \bar{\varphi}^{h}+\bar{p}_{o} \omega_{o}^{h}=0 \text { and } \bar{p}_{s}\left(\bar{x}_{s}^{h}-Y_{s}\left(\bar{x}_{o}^{h}+C \bar{\varphi}^{h}\right)\right)=\bar{D}_{s}\left(\bar{\theta}^{h}-\bar{\varphi}^{h}\right), s \in S \\
U^{h}\left(\bar{x}_{o}^{h}+C \bar{\varphi}^{h}, \bar{x}_{1}^{h}, \ldots, x_{s}, \ldots, \bar{x}_{S}^{h}\right) \geq U^{h}\left(\bar{x}_{o}^{h}+C \bar{\varphi}^{h}, \bar{x}_{-o}^{h}\right) \\
\Downarrow \\
\bar{p}_{s}\left(x_{s}+Y_{s}\left(x_{o}+C \varphi\right) \geq \bar{p}_{s}\left(\bar{x}_{s}^{h}+Y_{s}\left(\bar{x}_{o}^{h}+C \bar{\varphi}^{h}\right)\right)\right.
\end{gathered}
$$

Conditions (7) and (8) say that agents' asset holdings and consumption levels are minimizing expenditure, respectively, at date 0 and at date 1 in state $s \in S$. Thus we can claim that $\left(\bar{p}, \bar{\pi} ;\left(\bar{x}^{h}, \bar{\theta}^{h}, \bar{\varphi}^{h}\right)_{h \in H}\right)$ is a quasi-equilibrium for our exogenous collateral economy $E$.

\section{Step 2: From quasi-equilibrium to equilibrium}

Assumption (A4) and market clear conditions in both asset markets and first-period commodity markets imply that there is at least one agent $\bar{h} \in H$ for which

$$
\bar{D}_{s}\left(\bar{\theta}^{\bar{h}}-\bar{\varphi}^{\bar{h}}\right)+\bar{p}_{s} Y_{s}\left(\bar{x}_{o}+C \bar{\varphi}^{\bar{h}}\right)>0
$$

otherwise $\bar{p}_{s} Y_{s}\left(\sum_{h \in H} \omega_{o}^{h}\right) \leq 0$ contradicting (A4). This implies that $B_{s}^{\bar{h}}\left(p_{s}, x_{o}, \bar{\theta} \bar{h}, \varphi^{\bar{h}}, A_{s}, Y_{s}\right)$ is nonempty. Thus a standard argument implies that cost

14 Notice that in (4) and (6) the continuity of agents'utility functions has been used . 
minimization (9) implies utility maximization. That is, (6) holds in each state for $\bar{h}$ when $p_{s}^{n}=\bar{p}_{s}$ and $x_{n}^{h}=\bar{x}^{h}$. Strict monotonicity then implies that $\bar{p}_{-o}>0$

In what follows we prove that (4) and (5) remain held ${ }^{15}$ at $\left(\bar{x}^{h}, \bar{\theta}^{h}, \bar{\varphi}^{h}\right), \forall h \in H$. This together with (6') will imply that the quasi-equilibrium found is an equilibrium.

From (A6) it follows that at prices $\left(\bar{p}_{o}, \bar{\pi}\right)$ there is at least one agent $\bar{h}$ who can afford the portfolio $\left(\theta^{\bar{h}}, \varphi^{\bar{h}}\right)$ such that $\bar{p}_{o} \bar{x}_{o}+\bar{\pi} \theta^{\bar{h}}+\left(\bar{p}_{o} C-\bar{\pi}\right) \varphi^{\bar{h}}<\bar{p}_{o} \omega_{o}^{\bar{h}}$. Thus the interior of $B_{o}^{\bar{h}}\left(\bar{p}_{o}, \bar{\pi}, \omega_{o}^{h}, A, Y\right)$ is nonempty. Cost minimization (7) then implies utility maximization (4). Strict monotonicity again then implies that $\bar{p}_{o}>>, \bar{\pi}>>0$ and $\bar{p}_{o} C_{j}-\bar{\pi}_{j}>0, \forall j$.

Step 3: The non-emptiness of the interior of set $B_{o}^{h}$ for all agents

We will show that $\forall h \in H$, the interior of $B_{o}^{h}\left(\bar{p}_{o}, \bar{\pi}, \omega_{o}^{h}, A, Y\right)$ is nonempty. This ends the proof of the main theorem. (In fact), define the following sets

$$
\begin{aligned}
& H_{1}=\left\{h \in H: \operatorname{int} B_{o}^{h}\left(\bar{p}_{o}, \bar{\pi}, \omega_{o}^{h}, A, Y\right)=\phi\right\} \\
& H_{2}=H_{1}^{c}=\left\{h \in H: \operatorname{int} B_{o}^{h}\left(\bar{p}_{o}, \bar{\pi}, \omega_{o}^{h}, A, Y\right) \neq \phi\right\}
\end{aligned}
$$

From (A6) it follows that $H_{2}$ is a nonempty set. Therefore,

$$
\exists\left(x_{o}^{h}, \theta^{h}, \varphi^{h}\right): \bar{p}_{o} x_{o}^{h}+\bar{\pi} \theta^{h}+\left(\bar{p}_{o} C-\bar{\pi}\right) \varphi^{h}<\bar{p}_{o} \omega^{h}, \text { for some } h \in H_{2} .
$$

Suppose that the claim is false, that is $H_{1} \neq \phi$. Therefore the partition $\left\{H_{1}, H_{2}\right\}$ is not trivial. Then, from (A7) we can guarantee the existence of transfers $y=\left(y^{h}=\left(y_{o}^{h},\left(y_{s}^{h}\right)_{s \in S}\right)_{h \in H}\right.$ with $\sum_{h \in H} y^{h}=0$ and the existence of first-period consumption - investment $\left(\underline{x}_{o}^{h}, \underline{\theta}^{h}, \underline{\varphi}^{h}\right)$ such that

$$
\bar{p}_{s} y_{s}^{h}=\bar{p}_{s} Y_{s} \underline{x}_{o}^{h}+\bar{D}_{s} \underline{\theta}^{h}+\left(\bar{p}_{s} Y_{s} C-\bar{D}_{s}\right) \underline{\varphi}^{h}, s \in S ; \forall h \in H
$$

Furthermore,

$$
\forall h \in H_{1}, \omega_{o}^{h}+y_{o}^{h}, y_{s}^{h} \geq 0, s \in S
$$

15 That is, $\left(\bar{x}^{h}, \bar{\theta}^{h}, \bar{\varphi}^{h}\right)_{h \in H}$ are optimal choices for the respective agents. 
and

$$
\forall h \in H_{2}, U^{h}\left(x_{o}^{h}+C \varphi^{h}+y_{o}^{h}, x_{-o}^{h}+\left(y_{s}^{h}\right)_{s \in S}\right) \geq U^{h}\left(x_{o}^{h}+C \varphi^{h}, x_{-o}^{h}\right)
$$

with strict inequality for some $h \in H_{2}$.

We also know that $\left(\bar{p}, \bar{\pi} ;\left(\bar{x}^{h}, \bar{\theta}^{h}, \bar{\varphi}^{h}\right)_{h \in H}\right)$ is a quasi-equilibrium. Therefore from (10) and (11) it follows that

$$
\bar{p}_{o}\left(\bar{x}_{o}^{h}+\underline{x}_{o}^{h}\right)+\bar{\pi}\left(\bar{\theta}^{h}+\underline{\theta}^{h}\right)+\left(\bar{p}_{o} C-\bar{\pi}\right)\left(\bar{\varphi}^{h}+\underline{\varphi}^{h}\right) \geq \bar{p}_{o} \omega_{o}^{h}
$$

On the other hand, (A6) implies that $\left(\bar{x}^{h}, \bar{\theta}^{h}, \bar{\varphi}^{h}\right)$ maximizes the utility of agents of $H_{2}$. Thus, $\forall h \in H_{2}$,

$$
U^{h}\left(\bar{x}_{o}^{h}+C \bar{\varphi}^{h}+y_{o}^{h}, \bar{x}_{-o}^{h}+y_{s}^{h}\right) \geq U^{h}\left(\bar{x}_{o}^{h}+C \bar{\varphi}^{-h}, \bar{x}_{-o}^{h}\right)
$$

and

$$
\bar{p}_{s}\left(x_{s}^{h}+y_{s}^{h}\right)=\bar{p}_{s} Y_{s}\left(\bar{x}_{o}^{h}+\underline{x}_{o}^{h}\right)+\bar{D}_{s}\left(\bar{\theta}^{h}+\underline{\theta}^{h}\right)+\left(\bar{p}_{s} Y_{s} C-\bar{D}_{s}\right)\left(\bar{\varphi}^{h}+\underline{\varphi}^{h}\right), s \in S
$$

implies

$$
\bar{p}_{o}\left(\bar{x}_{o}^{h}+y_{o}^{h}\right)+\bar{\pi}\left(\bar{\theta}^{h}+\underline{\theta}^{h}\right)+\left(\bar{p}_{o} C-\bar{\pi}\right)\left(\bar{\varphi}^{h}+\underline{\varphi}^{h}\right)>\bar{p}_{o} \omega_{o}^{h}
$$

Summing among all agents of $H_{2}$ we have

$$
\bar{p}_{o} \sum_{h \in H_{2}}\left(\bar{x}_{o}^{h}+y_{o}^{h}\right)+\bar{\pi} \sum_{h \in H_{2}}\left(\bar{\theta}^{h}+\underline{\theta}^{h}\right)+\left(\bar{p}_{o} C-\bar{\pi}\right) \sum_{h \in H_{2}}\left(\bar{\varphi}^{h}+\underline{\varphi}^{h}\right)>\bar{p}_{o} \sum_{h \in H_{2}} \omega_{o}^{h}
$$

The following is true

$$
\sum_{h \in H}\left[\bar{x}_{o}^{h}+C \bar{\varphi}^{h}\right]=\sum_{h \in H} \omega_{o}^{h}, \sum_{h \in H} y_{o}^{h}=0 \text { and } \sum_{h \in H}\left(\bar{\theta}^{h}-\bar{\varphi}^{h}\right)=0
$$

$\sum_{h \in H}\left(\underline{\theta}^{h}-\underline{\varphi}^{h}\right)=0$. The last equality implied by (10) and by the fact that $\sum_{h \in H} y_{s}^{h}=0, s \in S$. Using these previous facts inequality (16)implies

$\bar{p}_{o} \sum_{h \in H_{1}}\left(\bar{x}_{o}^{h}+y_{o}^{h}\right)+\bar{\pi} \sum_{h \in H_{1}}\left(\bar{\theta}^{h}+\underline{\theta}^{h}\right)+\left(\bar{p}_{o} C-\bar{\pi}\right) \sum_{h \in H_{1}}\left(\bar{\varphi}^{h}+\underline{\varphi}^{h}\right)<\bar{p}_{o} \sum_{h \in H_{1}} \omega_{o}^{h}$ 
In consequence, there exists $h_{o} \in H_{1}$ such that

$$
\bar{p}_{o}\left(\bar{x}_{o}^{h_{o}}+y_{o}^{h_{o}}\right)+\bar{\pi}\left(\bar{\theta}^{h_{o}}+\underline{\theta}^{h_{o}}\right)+\left(\bar{p}_{o} C-\bar{\pi}\right)\left(\bar{\varphi}^{h_{o}}+\underline{\varphi}^{h_{o}}\right)<\bar{p}_{o} \omega_{o}^{h_{o}}
$$

In other words

$$
\operatorname{int} B_{o}^{h_{o}}\left(\left(\bar{p}_{o}, \bar{\pi}, \omega_{o}^{h}, A, Y\right) \neq \phi\right.
$$

which implies that $h_{o} \notin H_{1}$ which is a contradiction because $h_{o} \in H_{1}$. This contradiction, however, is caused for having supposed $H_{1} \neq \phi$. Finally $H_{1}=\phi$. Thus, the claim follows and the theorem as well.

\section{Conclusions}

In this paper we have demonstrated the existence of collateral equilibrium in a GEI model with default and collateral when the agents have no future income and the first-period social initial endowment is the boundary of the consumption set. Due to the fact that the first-period social initial endowment is not strictly positive, Geanakoplos and Zame's (2002) existence theorem cannot be applied.

The non satiated condition of the asset demand of consumers in this paper is implied by assumptions on collateral, assets structure and structure depreciation instead of being an hypothesis as in Gottardi and Hens (1996).

\section{References}

ARAÚJO, A.; FAJARDO, J.; PÁSCOA, M, R. Endogenous collateral. Journal of Mathematical Economics, v. 41, n. 4-5, p. 439-462, 2005.

GEANAKOPLOS, J. Promises, promises. In: ARTHUR, W.B.; DURLAUF, S.; LANE, D. (Ed.). The economy as an evolving complex system, II. Reading MA: AddisonWesley, 1997, p. 285-320. (CFDP 1143 and CFP 1057).

GEANAKOPLOS, J.; ZAME, W. Collateral and the enforcement of intertemporal contracts. 2002. (Yale University Working Paper).

GEANAKOPLOS, J.; ZAME, W.; DUBEY, R. Default, collateral, and derivatives. Yale University, 1995. Mimeo.

GOTTARDI, P.; THORSTEN, H. The survival assumption and existence of competitive equilibria when asset markets are incomplete. Journal of Economic Theory, v. 71, n. 2, p. 313-323, Nov. 1996. 
HILDENBRAND, W. Core and equilibria of a large economy. Princeton: Princeton University Press, 1974.

MCKENZIE, L. W. On the existence of general equilibrium for a competetive markets. Econometrica, v. 27, p. 54-71, 1959.

ORRILLO, J. Collateral once again. Economics Letters, v. 87, issue 1, p.27-33, April 2005.

SANDRONI, A. Do markets favor agents able to make accurate predictions? Econometrica, v. 68, n. 6, p. 1303-1341, Nov. 2000.

WERNER, J. Equilibrium in economics with incomplete financial markets. Journal of Economic Theory, v. 36, p. 110-119, 1985.

\section{Appendix A}

\section{A.1 Miscellaneous Notation}

Let $x \in R^{n}$ be a vector. We write $x>0$ to mean that $x \geq 0$ and $x \neq 0$. The vector $x_{-j} \in R^{n-1}$ is the vector $x$ where the $j$ - coordinate has been drooped. The same holds for bundles of vectors. If $x=\left(x_{1}, \ldots, x_{m}\right) \in R^{m n}$ then $x_{-k}$ is the bundle $x$ where the $k$ - th vector of $R^{n}$ has been drooped. By abuse of notation, we always use the same letter for both set and its cardinality. Thus, we will always write: $A=\{1, \ldots, A\}$. For $C=\left(C_{1}, \ldots, C_{J}\right) \in R^{J L}, C_{j} \in R^{L} ; \varphi \in R^{J}$ and $Y \in R^{L \times L}$, we set: $C \varphi=\sum_{j \in J} C_{j} \varphi_{j} \in R^{L}, Y C=\sum_{j \in J} Y C_{j} \in R^{L}$. For any two vectors $x, y \in R^{m}, x y=\sum_{i=1}^{m} x_{i} y_{i} \in R$ is the usual scalar product. Therefore, $\forall p \in R^{L}, p C \varphi=\sum_{j \in J} p C_{j} \varphi_{j}$.

The set $\Delta_{+}^{m-1}:=\left\{x \in R_{+}^{m}: \sum_{i=1}^{m} x_{i}=1\right\}$ is the non-negative simplex of $R^{m}$, and $\Delta_{++}^{m-1}=R_{++}^{m} \cap \Delta_{+}^{m-1}$ is the interior of the simplex $\Delta_{+}^{m-1}$. The map $\Pi_{i, j}: R^{m_{1}} \times \ldots \times R^{m_{k}} \rightarrow R^{m_{i}} \times R^{m_{j}}$ is the linear projection on the $i-t h$ and $j-t h$ factors defined as $\Pi_{i, j} x=\left(x_{i}, x_{j}\right) \in R_{i}^{m} \times R_{j}^{m}, x=\left(x_{1}, \ldots, x_{k}\right) \in R^{m_{1}} \times \ldots \times R^{m_{k}}$. If $M \in R^{m \times n}$ is a matrix of order $m \times n$, and $\beta \in R^{m}, y \in R^{n}$ are any two vectors, in the product $\beta M y \in R$, the vector $\beta$ will be a line vector and $y$ a column vector. Finally, $1 \in R^{n}$ is the vector whose coordinates are all equals 1 . 